\title{
Edición del videoclip y duración del plano: análisis del montaje de "I Took a Pill in Ibiza" (Mike Posner)
}

\section{Video clip editing and duration of the shot: analysis of the editing in 'I Took a Pill in Ibiza' (Mike Posner)}

José Patricio Pérez Rufí. Universidad de Málaga (patricioperez@uma.es)

Jennifer Rodríguez-López. Universidad de Huelva (jennifer.rodriguez@dedu.uhu.es)

Recibido: 11/07/2017 - Aceptado: 05/10/2017

\section{Resumen:}

El vídeo musical es un formato audiovisual creado generalmente por la industria discográfica con un objetivo comercial, promocional o conducente a la formación de la imagen pública de un intérprete musical. Esta investigación tiene como objetivo describir prácticas habituales en la realización y la edición del vídeo musical actual en un aspecto concreto: la duración del plano en función de su encuadre. Realizamos un análisis textual del videoclip de Mike Posner I Took a Pill in Ibiza (Jon Jon Augustavo, 2016). En este caso, los planos más frecuentes del videoclip son los planos cortos y sus duraciones medias están alrededor de los dos segundos, con una escala en duraciones medias paralela a la escala de los encuadres.

\section{Palabras clave:}

Videoclip; realización de televisión; montaje; postproducción audiovisual; análisis textual del discurso audiovisual.

\begin{abstract}
:
Music videos are an audiovisual format that is created by the music industry for a performer's public image and commercial purposes. This research aims to describe common practices in production and editing of current music videos regarding one particular aspect: the duration of the shot according to its frame. We carried out a textual analysis of Mike Posner's video clip I took a Pill in Ibiza (Jon Jon Augustavo, 2016). In this case, the most frequent shots in the video clip are close shots, and the average duration is around two seconds. The average length of the shots creates a scale of durations which is equivalent to the scale of the size of the frames.
\end{abstract}

\section{Keywords:}

Video clip, television production, editing, audiovisual post-production, textual analysis of audiovisual discourse. 


\section{Introducción}

Esta investigación pretende cuestionar la transgresión formal del videoclip en el campo de la realización y el montaje a partir del análisis de un aspecto concreto: la edición del videoclip desde el estudio de la duración del plano en función de sus encuadres. La permanente experimentación formal del formato y la voluntad por distanciarse de los códigos clásicos del discurso cinematográfico (e incluso televisivo) no explican solamente la dificultad que implica su modelización, sino también la descripción y la identificación de sus características definitorias.

Frente al videoclip, la principal característica que define la realización y la planificación en el cine clásico es la funcionalidad en la articulación del lenguaje audiovisual. En este, el diálogo entre forma y contenido propicia la subordinación de la planificación y los recursos discursivos a las necesidades dramáticas o narrativas del relato. Vernallis (2001: 22) sostiene que el vídeo musical, en el lado opuesto, hace del elemento narrativo solamente un parámetro más entre otros muchos destinados a captar nuestra atención. Según Jódar Marín, “a pesar de la innovación tecnológica aplicada al montaje y postproducción del videoclip, se sigue manteniendo su esencia como formato basado en la seducción para la promoción del producto musical que propone" (Jódar Marín, 2017: 126), aspecto que refuerza el componente discursivo del formato frente al narrativo.

Pérez Rufí et al (2014: 88) entienden a priori el videoclip como "una producción audiovisual condicionada por sus objetivos, generalmente comerciales y común en muchos aspectos a la publicidad televisiva, colindante formalmente con elecciones estéticas cercanas a la vanguardia y la experimentación", aunque subordinada a criterios de mercado que condicionarían los resultados. Sin embargo, la definición del vídeo musical no está exenta de dificultades, como concluyen Vernallis (2013: 181), Peverini (2002: 69) o Viñuela (2008: 236), razón por la cual lo entenderemos como un producto cultural en sí mismo (Sedeño, 2008), al servicio de los objetivos comerciales de la industria discográfica que, construido como creación audiovisual, "construye un relato, en la interrelación de música e imagen, que se confiere como una obra única” (Tarín Cañadas, 2012: 154).

Mantiene Sedeño que en el cruce entre medios audiovisuales y música popular, "el videoclip musical es un texto audiovisual híbrido, surgido de la interconexión o confluencia de una serie de tendencias tecnológicas, culturales y sociales en torno a la música popular" (Sedeño, 2010: 3). Roig y San Cornelio (2015: 51) reafirman esta naturaleza dual del videoclip, además cambiante a lo largo de su historia, tras haberse convertido "en uno de los principales campos de experimentación" tanto de fans como de otros colectivos.

Desde los años ochenta, señala Jones (2005), fue abundante la investigación en torno al formato, quedando en un segundo plano en la década de los noventa. Con el inicio del siglo, renace el interés por el vídeo musical de la mano de la notoriedad del trabajo de vídeo-creadores referenciales en el panorama audiovisual. Destacaremos los estudios de Vernallis 
(2013), Beebe y Middleton (2007), Cook (2000), Peverini (2002) o Austerlitz (2008) a nivel internacional y los de Sedeño (2008), Selva (2014) o Viñuela (2008) en la investigación nacional.

En relación con esta evolución de su investigación, apuntan Sedeño et al. (2016: 335-336) que "el videoclip ha sido objeto de numerosos acercamientos analíticos con el propósito de explicar su tránsito desde el medio televisivo hacia una producción con medios digitales y la distribución en Internet". Incluso si el análisis formal del videoclip no parece estar en la agenda de aquellas cuestiones de mayor interés en la investigación académica en comunicación, este merece ser atendido desde el momento en que cambia su contexto de distribución y consumo. La alta demanda de consumo del videoclip, recogida en los índices de contenidos con mayor cantidad de reproducciones de plataformas de vídeo online como YouTube, demuestra que no se trata de un formato menor en el conjunto de la producción audiovisual y que adquiere un protagonismo insólito en Internet, hecho que justifica su estudio como formato de encuentro y de cambio de los paradigmas de la nueva comunicación.

Como discurso de la fragmentación representativo de la post-modernidad (Rodríguez-López, 2016), el estudio de la velocidad del discurso en el videoclip no podría ser tratado como un baremo claro en cuanto a la fragmentación del discurso, afirma Pedrosa González (2016: 449), "ya que se trata de un dato engañoso que no llega a constatar aquellas partes del vídeo musical en las que se evidencia más la discontinuidad y la segmentación del mismo”. Sin embargo, como ocurre con el anuncio de televisión, la fragmentación no será solo un rasgo estilístico, sino también un medio para concentrar toda la información posible en un tiempo muy limitado (Rodríguez-López, 2016: 22).

Consideramos, y esa es la hipótesis que mantenemos en este trabajo, que el videoclip actualiza unas prácticas frecuentes en la edición que cuestionaría la libertad formal que se le supone a su lenguaje, al punto de permitir la identificación de unos indicios que apuntarían hacia una gramática del videoclip. Creemos así que la duración de los planos viene determinada por el tipo de encuadre o por la activación del movimiento de cámara, de tal forma que podemos esbozar unas primeras líneas para un intento de modelización y normalización del lenguaje del videoclip.

Puntualicemos, no obstante, que la búsqueda de una categorización o una modelización de los recursos estilísticos se nos antoja un objetivo de difícil logro para un artículo de investigación, por lo que optaremos por el estudio, el análisis y la modelización de la realización y la edición en una sola pieza. El siguiente paso, que no abordaremos aquí, sería contrastar esta categorización de recursos estilísticos con otras prácticas a fin de hacer extensivos o no dichas características. Esta investigación tiene así un carácter exploratorio en un área de muy compleja categorización que primará un análisis del lenguaje y de la técnica desde un plano formal y denotativo antes que el análisis de las sensaciones connotativas producto de la denotación del montaje. 


\section{Metodología}

Como formato audiovisual con gran variedad de opciones formales, el videoclip se filma y se edita a partir de una voluntad más artística, dejando en segundo plano con frecuencia la funcionalidad en el uso de la imagen, al tiempo que la narratividad tampoco se perfila como un objetivo. Esta variedad de opciones y la aparente ausencia de aplicación de una gramática de la imagen explican la complejidad de su estudio académico y los frustrantes intentos de categorización. Sostiene Sedeño (2008: 135) que el videoclip se caracteriza por "su tendencia a frustrar cualquier intento de categorización".

Tarín Cañadas (2013: 9) admite la complejidad del análisis del videoclip como formato audiovisual permeable al cambio "y al hibridismo de formas mediáticas", porque "se configura como una obra audiovisual en sí misma", aunque su primera vocación es la publicitaria. Su riqueza también estriba en su doble capacidad para llegar al espectador: "por la vía persuasiva a través de la espectacularidad; y/o por la vía narrativa con la creación de una historia que es contada a partir de una pieza musical" (Tarín Cañadas, 2013: 9).

La misma consideración del formato como paradigma de la postmodernidad (Rodríguez-López, 2016) nos aleja paradójicamente de una modelización que permita su definición, al menos desde criterios formales o desde la aplicación de recursos estilísticos precisos. Apunta, en todo caso, Fandos (1993: 95) que el clip musical configuró una "estética" particular con unos patrones propios, así como "acuerdos tácitos no escritos" que llevarían en último término a describir sus prácticas más habituales. Desde el momento en que hemos decidido focalizar nuestra atención en la práctica de sus opciones formales, nuestra investigación se articulará desde el análisis del discurso audiovisual y, dentro de este, el análisis textual.

Realizaremos, en este sentido, un análisis textual según entienden dicha metodología Casetti y Di Chio (1991: 33): hemos descompuesto las piezas presentes en el vídeo musical seleccionado (I Took a Pill in Ibiza Seeb Remix Explicit) para después recomponerlas y descubrir sus principios de construcción y su funcionamiento. Enunciado desde este planteamiento, el análisis textual se revela igualmente amplio y esquivo con nuestro preciso objeto de estudio: la edición del vídeo y la duración del plano. Nuestro enfoque nos invita a primar un análisis del discurso por encima del análisis de la historia o de la consideración de aspectos más narrativos.

Peverini (2002: 69-70) afirma que individualizar la puesta en escena del videoclip soportada desde el nivel discursivo es una forma interesante de análisis del formato. Separar el análisis discursivo (y, por tanto, enunciativo, añade), permitiría también comprender mejor la organización del nivel narrativo, así como aclarar la difusión de algunos estereotipos sobre la articulación de este tipo de texto. Además, sostiene que muchos videoclips no se conciben desde una estructura narrativa débil o ausente, fragmentando la puesta en escena (Peverini, 2002: 70), hecho que acreditaría la validez del análisis discursivo. 
Nuestra metodología de análisis no acude a los referentes de la narrativa audiovisual o lo hace de forma muy puntual: el interés de este trabajo remite a lo formal, a lo discursivo y al análisis técnico de la realización y la edición, antes que al estudio de la relación entre historia y discurso.

Esta investigación parte de la metodología y de los resultados obtenidos de un trabajo previo (Pérez Rufí y Rodríguez López, 2017) a partir del análisis de una muestra de videoclips representativos del contexto contemporáneo de producción. Así, hemos acudido a la lista Billboard de los singles más vendidos en Estados Unidos durante la semana del 9 de abril de 2016.

El éxito de estas producciones musicales son el resultado de una fuerte inversión de la industria discográfica en su promoción, pero también del reconocimiento y consumo de los clientes que adquieren contenidos musicales durante un breve periodo de tiempo, dado que la lista se actualiza semanalmente y refleja los productos más vendidos en la semana previa a la publicación de la lista. Consideramos además que los videoclips creados para la industria discográfica de los Estados Unidos suponen el mejor referente de la producción más comercial y mainstream.

En la investigación mencionada, podía establecerse la relación que recoge el Cuadro 1 entre duración de la pieza audiovisual (columna 2) expresada en minutos:segundos:frames, número de planos (columna 3) y duración media de cada plano (columna 4), expresada en segundos:frames. En dicho análisis se ha considerado la tradicional división de los códigos de tiempo del sistema de televisión PAL, en el que cada segundo de imagen de vídeo está formada por 25 frames. En cuestiones de códigos de tiempo y edición en España y Europa el referente sigue siendo el PAL, razón por la cual se mantienen estas unidades de tiempo y esta escala.

Cuadro 1: Tabla con duraciones, planos y medias de los videoclips de singles más vendidos en la lista Billboard (9/4/2016)

\begin{tabular}{|c|c|c|c|}
\hline Video & Duración & Número de Planos & Duración Media Planos \\
\hline Rihanna-Work (Explicit) ft. Drake & 03:44:15 & 126 & 01:19 \\
\hline Lukas Graham - 7 Years (Official Music Video) & $03: 58: 23$ & 123 & $01: 23$ \\
\hline Justin Bieber-Love Yourself (Purpose-The Movement) & $04: 31: 22$ & 30 & 09:01 \\
\hline Flo Rida - My House (Official Video) & 03:11:02 & 164 & 01:04 \\
\hline Twenty One Pilots - Stressed Out (Official Video) & 03:38:07 & 116 & $01: 22$ \\
\hline Meghan Trainor - No & 03:42:03 & 235 & 00:23 \\
\hline G-Eazy x Bebe Rexha-Me, Myself \& I & 05:47:21 & 214 & $01: 15$ \\
\hline Zayn-Pillowtalk & 03:26:09 & 119 & 01:18 \\
\hline DNCE - Cake by the Ocean & 04:17:01 & 166 & 01:13 \\
\hline Mike Posner-I Took a Pill in Ibiza (Seeb Remix) (Explicit) & 03:55:21 & 118 & 01:24 \\
\hline MEDIA & 04:01:20 & 141 & $01: 18$ \\
\hline
\end{tabular}

Fuente: Elaboración propia a partir de Pérez Rufí y Rodríguez-López (2017) 
Este trabajo concluye que el número medio de planos es de 141 por cada pieza. Observamos, sin embargo, que cinco de los vídeos rondan entre los 116 y los 126 planos, una horquilla de variación muy reducida si consideramos que las posibilidades podrían ser muy amplias en la muestra (desde los 30 planos del clip de Justin Bieber a los 235 de Meghan Trainor). Podríamos establecer que aunque la media real sería de 141 planos es más frecuente el videoclip con alrededor de 120 planos.

En esta ocasión iremos un paso más allá para analizar con detalle un solo vídeo musical. El análisis de un caso paradigmático puede cumplir con los objetivos de esta investigación, caso, por ejemplo, de Edmond (2012) con respecto a un videoclip de OK Go para comentar el cambio de paradigmas provocado por la distribución online del formato. Otros ejemplos podrían conformarlo el modelo de estudio de caso aplicado por Vernallis (2013) en el vídeo de Beyoncé y Lady Gaga "Video Phone" (Hype Williams); Tarín Cañadas (2012) realiza un análisis discursivo y narrativo de "Knives Out" de Radiohead (Michel Gondry); Roig y San Cornelio (2015) investigan el proceso de producción colectiva del proyecto "Evolution of Get Lucky" de PV Nova.

Martínez Carazo (2006: 189) afirma que el estudio de caso "es una estrategia metodológica de investigación científica, útil en la generación de resultados que posibilitan el fortalecimiento, crecimiento y desarrollo de las teorías existentes o el surgimiento de nuevos paradigmas científicos", siempre que se aplique el método con un adecuado diseño de la investigación y con rigurosidad científica. Creemos pues que el estudio de caso se erige como metodología válida para lograr conclusiones acerca de la creación y articulación del vídeo musical.

Apostamos por I Took a Pill in Ibiza (Seeb Remix) (Explicit) de Mike Posner como vídeo representativo de la realización del vídeo musical de producción estadounidense de 2016. Publicado en YouTube el 26 de febrero de 2016, el 10 de julio de 2017 había alcanzado las 824.794.305 visualizaciones en lo que supuso, sin lugar a dudas, uno de los vídeos más reproducidos en el portal de vídeo de Google en el año 2016. Fue dirigido por Jon Jon Augustavo, prolífico realizador con 53 clips bajo su firma creados entre 2010 y 2017, según recoge la base de datos The Internet Music Video Database (2017). La pieza fue nominada al premio Best Electronic Video de los MTV Music Awards (edición estadounidense) y al Premio a Mejor Canción (edición europea) y está disponible a través de YouTube (Posner, 2016).

Consideraremos en cada uno de los planos el tamaño del encuadre, donde seguiremos la tradicional escala europea de encuadres, adaptando las clasificaciones de Villafañe (2006) y Millerson (1991): Gran Plano General (GPG); Plano General (PG); Plano Entero (PE); Plano Americano (PA); Plano Medio Largo (PML); Plano Medio (PM); Plano Medio Corto (PMC); Primer Plano (PP) y Plano Detalle (PD). Apuntemos que hemos incluido el Primerísimo Primer Plano dentro del mismo encuadre que el Plano Detalle. En el caso de que el plano incluya diversos encuadres, por ejemplo en un movimiento de cámara (travelling, zoom, movimiento digital), hemos tomado nota de dichos cambios, pero en el cuadro de resultados hemos optado por indicar el tamaño del encuadre con que finaliza el plano. 
Junto a los diversos tipos de encuadres, hemos añadido una categoría de análisis denominada "cartón" (en el cuadro 2 indicado como "Cart"), que sería un plano formado por una imagen fija, por lo general generada por grafismo digital: un plano en negro, un plano con rotulación o un plano con un color sólido o solo con rotulación, es decir, con elementos de grafismo. Contabilizamos el número de planos según cada encuadre para llegar a los porcentajes medios de presencia de cada tipo de encuadre y sus duraciones. Durante el proceso de análisis hemos anotado la duración de cada plano.

A continuación atendemos a la posibilidad de cambio de encuadre limitándolo a tres posibles opciones: movimiento de cámara (travelling) (en el cuadro 2, "Trav"), movimiento de óptica zoom (en el cuadro 2, “zoom”) o re-encuadre digital en el mismo plano (no representado en el cuadro 2).

En tercer lugar, observamos la relación entre la duración del plano y la duración de la acción representada para identificar si esta es normal, ralentí (en el cuadro 2, "Ral”), acelerado (en el cuadro 2, "Acel”) o si, a lo largo del mismo plano, hay variaciones en cuanto a la aceleración.

En cuarto lugar, tomamos nota de los tipos de transición que se producen entre los planos (no recogido en el cuadro 2, dado que el $100 \%$ de las transiciones son al corte). Como efecto particular del cambio de plano, anotaremos aquellas transiciones en las que el cambio por corte provoca un efecto de jump cut (o de fallo de rácord por mantenimiento de encuadre y de motivos entre planos separados por una transición al corte, no recogido en el cuadro 2).

\section{Resultados y discusión}

Hemos realizado en primer lugar una tabla en la que recogemos los datos relativos a cada uno de los 118 planos en cuanto a tipo de encuadre, uso de "cartones", movimiento de cámara, aceleración o ralentización del plano y efecto en la transición. No hemos incluido dicha tabla en esta publicación debido a su extensión, pero a partir de esta hemos elaborado esta otra tabla que resume sus principales aportaciones. En dicha tabla (cuadro 2) representamos la relación de planos, suma de tiempos totales de cada tipo de plano y duración media del plano en función del encuadre. De nuevo volvemos a expresar los valores del total de tiempos y de duración media por plano en minutos:segundos:frames (mm:ss:ff) o en segundos:frames (s:ff). 
Cuadro 2: Tabla con número total de planos según cada tipo y duraciones totales y medias por tipo de encuadre

Mike Posner - I Took a Pill in Ibiza (Seeb Remix) (Explicit). Director: Jon Jon Augustavo (2016)

\begin{tabular}{|c|c|c|c|c|c|c|c|c|c|c|c|c|c|c|}
\hline Tipo de plano & GPG & PG & $\mathrm{PE}$ & PA & PML & PM & PMC & PP & PD & Cart & Trav & Zoom & Ral & Acel \\
\hline Total de planos & 0 & 0 & 4 & 8 & 3 & 32 & 8 & 26 & 34 & 3 & 26 & 0 & 64 & 13 \\
\hline Total duración & 0 & 0 & 9:18 & 18:12 & 11:04 & 1:13:01 & $16: 24$ & 43:21 & $53: 16$ & 7:07 & 1:15:24 & 0 & $2: 05: 15$ & $24: 10$ \\
\hline $\begin{array}{l}\text { Duración media / } \\
\text { planos }\end{array}$ & 0 & 0 & $2: 10$ & $2: 07$ & $3: 18$ & $2: 07$ & 2:03 & $1: 17$ & $1: 14$ & $2: 10$ & $2: 23$ & 0 & $1: 24$ & $1: 21$ \\
\hline
\end{tabular}

Fuente: Elaboración propia

\subsection{Fase previa al análisis: duración de la canción y del videoclip}

Comentaremos, en primer lugar, que la duración del videoclip no coincide, como venía siendo habitual en el formato, con la duración de la canción. Así, mientras que el clip dura 03:56 (minutos:segundos), la canción llega solo hasta los 03:17 (minutos:segundos). A diferencia de los canales de televisión convencional (con una programación lineal y una edición muy controlada), YouTube no limita la duración de las piezas audiovisuales, con lo cual esta puede extenderse y no hacer coincidir la duración del videoclip con la del single editado. Ello permite un recurso en desuso desde la década de los noventa: el inicio del clip con una introducción narrativa, con recursos absolutamente cinematográficos, que ubicarán la acción y los personajes dentro del relato.

En los 39 segundos de introducción del vídeo, antes del inicio de la canción, se suceden algo más de 4 segundos de un plano fijo con la rotulación indicando el nombre de la canción y del músico, al que sigue una pequeña secuencia de apenas 30 segundos donde se presenta al intérprete en unos baños públicos consumiendo una pastilla de éxtasis, sorprendido ante los efectos que le provoca. La sorpresa se mezcla con la confusión, como representaría el uso de subtítulos en lenguas orientales que suponemos desconocidas para el personaje. Como banda de sonido, además de la voz del personaje, se oye en segundo plano un tema de música house que nos invita a pensar que está en una discoteca, como después se confirma. La canción es un tema del mismo artista, Mike Posner, In The Arms Of A Stranger (Brian Kierulf Remix), en un ejercicio de auto-referencialidad o de uso promocional del clip para otros contenidos propios.

En cuanto al concepto de la pieza, como el propio título de la canción indica, narra la experiencia del intérprete tras ingerir una pastilla en una discoteca ibicenca. El estado que le provoca la droga, desde una visión crítica contra su consumo, se describe en la alucinación descrita por la secuencia de planos del vídeo musical. Es la alteración de los sentidos bajo la intoxicación la que explicaría no ya solo la caracterización del personaje, con una máscara con los ojos dilatados, sino también un montaje acelerado de planos donde en ocasiones se alternan planos con cambios de aceleración en la reproducción, saltos de montaje o constantes movimientos de cámara. 
De esta forma, el discurso pretende representar formalmente el estado interior del personaje, además en consonancia con la letra de la canción. El vídeo musical, en líneas generales, no tiene por qué identificarse con la letra del tema musical, pero en este caso sí ocurre así. Esta cohesión entre forma y contenido explicará en gran medida la elección de muchos recursos relacionados con el discurso, o con la realización y la edición del vídeo, para ser más precisos.

\subsection{Duraciones medias de los planos}

La duración media de los 118 planos que se desarrollan en los 3 minutos con 56 segundos del videoclip (en realidad 3:55:21, a falta de 4 frames para llegar a completar el minuto 3:56) sería de 1:24, al borde de los 2 segundos de duración. Como en las tablas anteriores, expresamos los códigos en segundos y frames (s:ff). Tenemos así una media de escasísima duración que provocaría una enorme velocidad en el cambio de plano y la consecuente impresión de ritmo. En todo caso, este valor medio es muy cuestionable y no debería invitarnos a pensar que todos los planos tienen la misma duración.

Por el contrario, podemos apreciar desde un Plano Medio en ralentí con movimiento travelling (el número 13) con una duración de 9:17, hasta una secuencia formada por planos que no llegan a los 10 frames; en concreto el plano más breve es un Plano Detalle de 7 frames (plano 45). De los 118 planos que conforman el clip, 24 (esto es, un 20.3\%) no llegan al segundo de duración. Estos planos serían los números 30, 31, 32, 33, 38, 39, 40, 41, 44, 45, 77, 85, 86, 89, 90, 92, 103, 104, $105,107,108,110,113$ y 115.

Observamos que, incluso si una de las supuestas normas del videoclip es la ausencia de normas, los planos de menor duración se agrupan para crear una impresión de secuencia completa de mayor ritmo, logrando así una coherencia dentro del ritmo, que viene a coincidir con el estribillo de la canción. Salvando el plano 77, aislado y con una duración de 19 frames, el resto de planos de 14-15 frames se agrupan en alrededor de 4 secuencias de mayor velocidad, con hasta 4 planos sucesivos de entre 13 y 15 frames. Los planos de menor duración se mueven en la escala de encuadres entre el Plano Medio y el Plano Detalle, coincidiendo con el mayor porcentaje de encuadres presentes.

Destaquemos también que los planos de menor duración se concentran en la última parte del clip, en concreto entre los planos 85 y 115. La edición adquiere así una estructura a partir de una progresión en la duración del plano, cada vez menor, de donde resulta un crecimiento del ritmo en la sucesión de planos. Tras el "bombardeo" de planos que se produce entre los planos 103 y 115 (con la excepción del "largo" plano 114 (Plano Americano en ralentí con travelling), los tres último planos rompen el ritmo con planos de más de 3 segundos y más de 5 segundos, para terminar con una imagen fija en negro con el nombre del realizador de algo más de 2 segundos, que hemos contabilizado en el total como parte del clip.

La edición tiende a acortar cada vez más los tiempos en los planos e incrementarse a modo de clímax en sus últimos segundos, para frenar bruscamente con planos más largos en duración que anuncian la conclusión de la pieza. La brevedad en los planos puede explicarse desde el momento en que el videoclip, con mucha frecuencia, hace uso de la repetición 
de planos y encuadres como recurso habitual: el espectador ya ha decodificado e interpretado previamente los planos y sus encuadres y el discurso puede permitirse limitar aún más la exposición de cada uno de los planos si aboga por la repetición de aquellos o de sus motivos.

Las investigaciones previas acerca de la duración del plano han destacado la articulación de la secuencia y la construcción de las unidades internas de los mensajes o en los valores de tiempo asignados habitualmente para la duración de los planos en el montaje de secuencias (Millerson, 2001; Sánchez, 1996), además de diversos experimentos que dan cuenta del umbral de percepción visual y auditiva.

Aunque el objeto de esta investigación no es indagar en los umbrales de percepción, destacaremos tres ideas de Morales Morante (2009: 162-163): la identificación de formas y de los elementos que componen el encuadre se distinguen en función de un proceso selectivo según su relevancia, coherencia o predominio; la repetición facilita el reconocimiento; la identificación de la escena requiere una base mínima de perceptibilidad del estímulo, lo que nos lleva de nuevo a los límites de los umbrales de percepción.

\subsection{Frecuencia de encuadres y relación con la duración del plano}

Como ya hemos avanzado, los tipos de encuadres más habituales serían los planos cortos. Los planos más frecuentes son los Planos Detalle (28.8\%), Planos Medios (27.1\%) y Primeros Planos (22\%). Si sumamos los 8 Planos Medios Cortos (6.7\%), tendríamos que el $84 \%$ de los planos (100 de 118) realizaría encuadres cortos con poquísima variedad en la escala de planos. Apenas 15 planos (el 12.7\%) superan el Plano Medio con encuadres que van del Plano Medio Largo al Plano Entero, estando por completo ausentes los encuadres superiores.

La realización es eminentemente televisiva y los encuadres se ocupan así con motivos que llenan la pantalla y permiten focalizar la atención en los personajes, en la representación de sus posibles emociones y en sus acciones antes que en la descripción del entorno o en la contextualización de la acción que permitirían planos más amplios. No necesitamos Planos Generales para comprender que el protagonista del videoclip está en una discoteca; por el contrario, el interés está en su vivencia de la experiencia del consumo de drogas, incluso si la máscara que cubre su rostro muestra el mismo gesto de forma permanente.

En todo caso, los planos que permitirán una descripción del contexto espacial y del resto de clientes del local de ocio son Planos Enteros y Planos Medios Largos, apropiados para lograr dicha representación del entorno en un espacio que entendemos no es muy amplio. La combinación de diversos recursos de la puesta en escena, como el uso de planos cortos, una luminosidad limitada, coloreada y basada en el uso de luces estroboscópicas en ocasiones, además de la acumulación de personajes/cuerpos cercanos y en contacto, provocarán una impresión claustrofóbica o de encierro. La ingestión 
de la pastilla alucinógena encierra así al protagonista en un espacio interior y oscuro, que se puede interpretar como una representación externa de su experiencia interior.

No conocemos el total de audiencia que ha podido acumular este videoclip en sus diferentes pases en televisión, pero sí sabemos que más de 824 millones de usuarios de YouTube visualizaron el clip a través de la plataforma de contenidos de vídeo de Google. El vídeo contó con una distribución multiplataforma en televisión y online mediante plataformas como YouTube. Si Internet y YouTube fueran el medio preferente de distribución de este clip, podríamos deducir que se ha abogado por el uso de planos cortos para crear una experiencia de visionado adecuada a las condiciones que impone el medio, donde el objetivo volvería a ser, si acaso de forma más extrema que en la televisión convencional, "llenar la pantalla".

Los usuarios de YouTube, como marca multiplataforma, consumen sus contenidos en pantallas cada vez más amplias, pero también en dispositivos móviles y portátiles con pequeñas pantallas en las que conviene llenar los encuadres. La apuesta por el plano corto que hace "I Took a Pill in Ibiza" garantiza la atención y la tensión que generan los planos cortos, sin optar por los encuadres más amplios, más arriesgados consecuentemente.

Si atendemos a las duraciones medias de cada tipo de encuadre nos sorprenderá descubrir que no hay variaciones sustanciales entre ellas. En una realización clásica, acorde con los principios del MRI (Modelo de Representación Institucional) (Burch, 2006) que después heredó la televisión, los encuadres más amplios solían requerir una mayor duración, un mayor desarrollo, por decirlo de otra forma, para poder permitir al espectador una exploración del cuadro completo (Pérez Rufí, 2009: 108-109).

De manera paralela, los encuadres más cortos eran explorados e interpretados con mayor rapidez, lo que permitía la posible reducción de la duración del plano. Maticemos, en todo caso, que la atención y la permanencia del encuadre en la pantalla vienen determinadas por muchos factores, no solo por el tamaño del encuadre, sino, de forma muy especial, por el contenido mostrado en el encuadre, por la acción representada o por la tensión que provoca el diálogo, la música o cualquier otro recurso.

El videoclip analizado presenta valores bastante similares en sus duraciones. Así, la duración media de los Planos Enteros es de 2:10, la de los Planos Americanos 2:07 y los Planos Medios Largos 3:18, códigos muy cercanos a la media inferior a los 2 segundos que señalábamos. Los Planos Medios coinciden en duración media con los Planos Americanos (2:07) y, si bien la duración del resto de planos cortos es ligeramente inferior, estamos hablamos de duraciones de entre 15-20 frames menos: la media de los Planos Medios Cortos es de 2:03, los Primeros Planos 1:17 y los Planos Detalles 1:14.

La interpretación que hacemos de estos datos no puede ser contundente. Podríamos evaluar estas medias como similares y poco significativas, por lo que no identificaríamos un claro código que implicara una mayor duración de los encuadres mayores y una duración menor de los cortos, pero también podríamos acentuar el valor de las pequeñas matizaciones y 
diferencias, no ya de segundos sino de frames, para reforzar la idea de que, efectivamente, hay una escala en la duración de los planos equivalente al tamaño del encuadre.

Los Planos Enteros duran una media de 2:10, mientras que los Primeros Planos bajan a 1:17 y los Planos Detalles aún reducen esta duración 3 frames más para llegar a los 1:14. De alguna forma, aun con la suficiente precaución, podríamos confirmar que en el videoclip analizado queda algo de aquella norma ligada a un código más clásico que vincula duraciones con tipos de encuadres.

\subsection{Duraciones medias de los encuadres más amplios}

Procedemos a continuación al comentario de cada tipo de encuadre, habida cuenta de la variedad que ofrece y de las matizaciones que introducen con respecto a las duraciones medias. Como ya hemos apuntado, los Grandes Planos Generales y los Planos Generales están ausentes en la pieza analizada, quedando así fuera un tipo de encuadre funcional en la descripción de entornos, la creación de tomas espectaculares desde puntos de vistas insólitos para el espectador (como hacen las tomas aéreas) o la presentación de multitudes o de grandes espacios abiertos. Como ya mencionamos, la permanente ubicación de la acción en espacios interiores de escasa luminosidad "llama” al uso de otros encuadres más cortos.

Los Planos Enteros presentes en el videoclip son muy pocos, apenas 4 (un 3.3\% del total de planos), además editados en continuidad (son los planos 26, 27, 28 y 30). Sus duraciones (con una media de 2:10) son muy variadas, yendo de los 5:10 del plano 28 (es el tercer plano más largo en duración de la pieza) a los 14 frames de la secuencia de 4 planos editados en continuidad con una duración de 14 frames (15 en el caso del plano 33). Los otros dos planos se mueven en la media de la duración media de todos los planos, 1:18 (plano 26) y 1:23 (plano 27).

Estos valores tan variados en una muestra tan escasa de Planos Enteros no nos permitirían generalizar ni extraer conclusiones lo bastante contrastadas, pero sí que podemos llegar al menos a la idea de que no hay un patrón determinado en cuanto a duraciones de los Planos Enteros.

El número de Planos Americanos sube hasta 8, con una duración media de 2:07. Este tipo de planos aparece de forma dispersa por todo el plano y no se editan en continuidad, al ir precedidos o seguidos de Planos Medios o Planos Detalle. El paso de planos amplios a planos cortos incrementará el ritmo del discurso gracias al aumento de la tensión que ocasiona, produciéndose el efecto inverso, la deceleración, en la transición de planos cortos a planos más amplios (Pérez Rufí, 2009: 110).

De esta forma, incluso si la diferencia entre planos no es extrema, se juega con la aceleración y la desaceleración del ritmo para mantener la atención del espectador. Las duraciones vuelven a ser muy variadas, entre los 6:07 del plano 75 (el segundo más largo de todo el videoclip) hasta los 14 y los 15 frames de los planos 89 y 92 . El resto de Planos Americanos no sigue tendencias mayoritarias, ya que alternan los 4:05 (plano 14), 2:23 (plano 64), 1:15 (plano 35) o 1:04 (planos 73 
y 106). La función del Plano Americano es similar a la del Plano Entero, si bien es más frecuente al acercarse más a los planos más cortos.

Esta variedad en la duración del Plano Americano podría interpretarse como una forma de evitar la repetición en las duraciones de los planos y así crear un ritmo monótono, como ocurría con el recurso del corte coincidente con el golpe rítmico de la percusión del tema musical, tan frecuente en los clips de los años ochenta (la edición denominada cut to the beat). Liao et al. (2015: 1-2) amplían este sentido del cut to beat al corte sobre cualquier nota significativa y la sincronización entre vídeo y música a partir del ritmo de la música.

Con solo tres Planos Medios Largos, la variedad de planos es tan escasa que todas las conclusiones serían por fuerza parciales. Los planos con este encuadre localizados (planos 3, 11 y 116) duran respectivamente 5:07, 2:17 y 1:19, que explican los 3:18 de duración media, el valor más alto de todas las medias por tipo de encuadre.

\subsection{Duraciones medias de los encuadres más cortos}

Los Planos Medios son los segundos más frecuentes después de los Planos Detalles, presente en 32 ocasiones y conformando un 27.1\% del total de planos del vídeo analizado. Con una duración media de 2:07, encontraremos, sin embargo, una enorme variedad de duraciones, por lo que resultará complejo llegar a conclusiones que relacionen el encuadre con la duración. El plano más largo de toda la pieza, como ya comentamos, es un Plano Medio (plano 13) con una duración de 9:17, en ralentí y con un movimiento travelling.

Otras duraciones superiores a la media las encontramos en los plano 18 (5:20), 51 (4:08), 67 (3:12) y 117 (5:08). El resto se moverá entre el segundo y los 2 segundos, si bien, como ya mencionamos, entre los planos 38 y 41 se suceden cuatro Planos Medios en ralentí con montaje al corte que provoca un efecto de jump cut (salto de montaje). Se trataría de la secuencia que ya comentamos de planos de brevísima duración que se editan en sucesión para crear un efecto de ritmo acelerado, pero también de salto de montaje entre planos. La repetición de encuadres y de similares motivos en cuatro planos consecutivos permite la correcta exploración de la imagen y crean una unidad rítmica dentro de la pieza.

Hemos destacado dentro del Plano Medio aquellos que exceden o no llegan a la duración media, pero hemos de advertir que al menos 23 de los 32 Planos Medios duran entre 1 y 2 segundos, lo que explica tanto la duración media del Plano Medio como la media de todos los planos del videoclip que, como mencionamos, ronda los 2 segundos.

El Plano Medio Corto tiene una duración media de 2:03, con 8 apariciones en todo el discurso. De la misma forma que este tipo de encuadre ofrece un tamaño del personaje entre el Plano Medio y el Primer Plano, la media en la duración también estaría entre la media del Plano Medio (2:07) y la del Primer Plano (1:17), hecho que se encuadra dentro de la supuesta 
escala de encuadres y duraciones que ya destacamos. Los 8 Planos Medios Cortos de la pieza (un 6.7\% del total) varían en sus duraciones entre un segundo y 4 segundos, sin que detectemos tampoco tendencias o valores más frecuentes.

Los Primeros Planos conforman un 26\% del total de planos de la pieza (22 planos) y reducen apenas unos frames la media de los Planos Medios y los Planos Medios Cortos, con lo que se confirma la práctica habitual de reducir los tiempos de los planos más cortos. La diferencia de duraciones vuelve a ser contundente pero, en todo caso, los planos de mayor duración no superan los 4:23. El más breve apenas llega a las 7 frames y, como en el Plano Medio, los valores más frecuentes se mueven entre el segundo y los 2 segundos, con algunas ráfagas de planos de 14-15 frames.

Siguiendo la gramática audiovisual más clásica, el Primer Plano permite centrar la atención en la descripción de la emoción del personaje, como ya comentamos con respecto a los planos cortos. Será el mismo caso de los Planos Detalles (y del Primerísimo Primer Plano), el más frecuente del discurso con 34 apariciones (un $28.8 \%$ del total de planos). La media vuelve a ser inferior al del plano superior en la escala, 1:14 y, en este caso, no existe una diferencia muy marcada entre los Planos Detalles de mayor duración y de menor duración, dado que el más breve dura 15 frames y más largo 2:22. Encontramos así una mayor coherencia en los Planos Detalles, vinculados a duraciones más breves, por tanto.

\subsection{Duraciones de las imágenes fijas}

Apuntemos que las tres imágenes fijas ("cartones") con elementos de grafismo (rotulación) tienen una duración de 4:03 y 2:18, duración suficiente para la lectura de las palabras representadas, además de un plano en negro con una duración de 11 frames que actúa a modo de separador en la conclusión del vídeo. Este tipo de planos es puramente funcional, al quedar al servicio de la transmisión de una información textual y actuar como introducción y cierre de la pieza.

\subsection{Duraciones medias de los planos con movimiento de cámara}

Con respecto a los movimientos de cámara, contabilizamos 27 planos que lo aplican ( $22.8 \%$ del total), de los que 26 son travellings o movimientos físicos de la cámara, un solo plano hace movimiento óptico de lentes (zoom) y no hay, aparentemente, movimientos de re-encuadre realizados digitalmente. Se impone así en este videoclip un modo más tradicional del movimiento de cámara evitando recursos asociados a la televisión de décadas atrás como es el zoom.

En lo relativo a la duración de estos planos, cae en este caso la idea de que el travelling invita a planos de mayor duración, dado que la duración media es de 2:23. Es en la edición de este tipo de planos que el vídeo musical reivindica su carácter disruptivo y rompe con convenciones del MRI. Destaquemos, en todo caso, la idea de que los planos más largos de la pieza (con duraciones de 9:17, 6:07 o 5:07) son planos con travelling, dato que podría reafirmar la idea del travelling asociado a una mayor duración. 


\subsection{Otros efectos de edición: cambios de aceleración y transiciones}

Los juegos con la aceleración y desaceleración dentro del mismo plano fueron un recurso habitual en el videoclip de los noventa, después en desuso por sobreexplotación. Los planos en ralentí, que aquí conforman un 54.2\% del total, "poetizan" los resultados, en el sentido de que se pierde la relación con la reproducción normal (coincidente con la duración real de la acción representada) y conforma así un entorno irreal marcado por la suavidad en la realización de las acciones. La duración media de los planos en ralentí es también muy breve (1:24), con lo cual las acciones se descomponen aún más al fragmentarse su desarrollo en el tiempo. Frente a la alta frecuencia del plano en ralentí, el plano de aceleración superior a la normal hace aparición solo en 13 planos (un 11\% del total), por lo que no llega a abusarse de un recurso mucho más agresivo que el ralentí.

Como ya comentamos, todas las transiciones son al corte, de forma que el cambio de plano es más enérgico y duro que con otros modos de transición. Se exagera esta intensidad del corte en aquellos planos en los que se mantienen los encuadres tras el corte, produciéndose un salto de montaje. En esta pieza se produce en siete ocasiones, por lo general en planos de duración muy breve (14-15 frames) consecutivos, lo que permite respetar la creación de un ritmo y la exploración correcta del encuadre. Aunque es un recurso de montaje muy explotado en los clips de las dos últimas décadas y ha perdido su frescura, mantiene su vigencia en el videoclip contemporáneo.

\section{Conclusiones}

Las conclusiones a las que lleva nuestro análisis son por fuerza parciales y relativas a la pieza objeto de nuestro análisis, lo que no aconsejaría extrapolar los resultados a la construcción de todos los videoclips. Sin embargo, un estudio detallado de una sola pieza nos permite contrastar algunas de las ideas a priori atribuidas al vídeo musical en lo relativo a realización y edición.

Así, en el caso que nos ocupa se mantiene la asociación del vídeo musical con la idea de sucesión de planos con muy breve duración, como demuestra la duración media del total de planos, así como las duraciones medias por tipo de encuadre. El principio propio de un lenguaje audiovisual más clásico que vincula los planos de mayor tamaño con unas duraciones superiores no llega a confirmarse por completo en el clip analizado, si bien descubrimos que sí existe una escala de duraciones paralelas a los encuadres, de tal modo que, atendiendo a las medias, los planos más cortos tendrán un desarrollo más breve en el tiempo, apenas unos frames menos. Esta conclusión refuerza la idea de que los encuadres más cortos no requerirían una mayor duración, razón por la cual en la práctica los editores han optado con frecuencia por acortar su desarrollo en el tiempo, llegando al extremo en un formato supuestamente abierto a la transgresión formal como es el videoclip. 
Incluso si no hemos realizado un análisis de la narración ni de los efectos connotativos de las elecciones de montaje, podemos observar que la experiencia vital de un personaje condicionada por la ingesta de sustancias alucinógenas conduce a una elección de planos que prioriza la descripción del protagonista y de sus emociones por encima de la descripción del entorno y del desarrollo de acciones. Ello explicaría la preferencia por encuadres cortos, pero también otros recursos como el cambio de aceleración en la reproducción de los planos o los saltos de montaje, dada la visión distorsionada de la "realidad" del personaje. Con todo, el videoclip puede permitirse este tipo de recursos de edición sin necesidad de una motivación desde el relato representado.

La suma de planos de muy breve duración con encuadres cortos, cercanos al personaje, provoca una doble fragmentación en la representación de las escenas: por una parte, el encuadre se fragmenta al recurrirse a planos cortos (entre Planos Medios y Planos Detalles, en su mayoría); por otra parte, el tiempo se fragmenta a partir del breve desarrollo de cada plano. La fragmentación se convierte así en una característica definidora del vídeo musical, conclusión que podríamos ampliar al conjunto del formato.

Concluimos que no podemos "medir" el nivel de fragmentación dada la enorme variedad de posibilidades que ofrece el vídeo, como vemos en la disparidad de duraciones de los planos. Podemos atisbar, en todo caso, unos mínimos niveles de formalidad o de clasicismo discursivo que garantizarían una transmisión de un mensaje narrativo e incluso de un relato. Será pues la posible narratividad del discurso analizado la que obligue o no al seguimiento de un código de contrastada funcionalidad como la del lenguaje clásico (MRI). En todo caso, la duración del plano queda subordinada al efectismo del montaje, por lo que nuestro trabajo puede concluir. Una ampliación de esta investigación invitaría al contraste de los aspectos cuantitativos que aporta este trabajo con otros cualitativos, a fin de lograr un análisis de las sensaciones connotativas producto de la denotación del montaje.

\section{Referencias bibliográficas}

Austerlitz, S. (2008): Money for Nothing. A History of the Music Video from the Beatles to the White Stripes. Londres: Continuum.

Beebe, R. y Middleton, J. (2007): Medium Cool: Music Videos from Soundies to Cellphones. Durham: Duke University Press Books.

Burch, N. (2006): El tragaluz del infinito. Madrid: Cátedra.

Casetti, F. y Di Chio, F. (1991): Cómo analizar un film. Barcelona: Paidós.

Cook, N. (2000): Analysis Musical Multimedia. Oxford: Oxford University Press. 
Edmond, M. (2012): “Here We Go Again: Music Videos after YouTube”, Television \& New Media 2014, vol. 15, n. 4, pp. 305320. Disponible en Internet: http://journals.sagepub.com/doi/10.1177/1527476412465901 [Consultado el 07/07/2017].

Fandos Igado, M. (1993): “Una asignatura pendiente. El videoclip musical”, Revista Comunicar, no 1, pp. 94-97. Disponible en Internet: www.agifreu.com/docencia/lectures_obligatories/comunicar.pdf [Consultado el 07/07/2017].

Internet Music Video Database (2017): “Jon Jon Augustavo”. Disponible en Internet: https://imvdb.com/n/jon-jon-augustavopdf [Consultado el 07/07/2017].

Jódar Marín, J.A. (2017): "Evolución del montaje y postproducción del videoclip musical: del jumpcut a los VFX como paradigma de iconicidad y puesta en escena", Revista Mediterránea de Comunicación, vol. 8, nº 2. Disponible en Internet: https://rua.ua.es/dspace/bitstream/10045/67632/1/ReMedCom_08_02_11.pdf [Consultado el 07/07/2017].

Jones, S. (2005): “MTV: The Medium as the Message”, Critical Studies in Media Communication, n. 22, pp. 83-88. Disponible en Internet: http://stevejones.me/pubs/2005/CSMC-MTV.pdf [Consultado el 07/07/2017].

Liao, Z., Yu, Y., Gong, B. y Cheng, L. (2015): “Audeosynth: music-driven video montage”, Journal ACM Transactions on Graphics, vol. 34, n. 4. Disponible en Internet: http://hdl.handle.net/10722/215520 [Consultado el 07/07/2017].

Martínez Carazo, C. P. (2006): "El método de estudio de caso: estrategia metodológica de la investigación científica", Pensamiento y Gestión, 20, 165-193. Disponible en Internet: www.redalyc.org/pdf/646/64602005.pdf [Consultado el 07/07/2017].

Millerson, G. (1991): Técnicas de realización y producción en televisión. Madrid: Instituto Oficial de Radio Televisión.

Morales-Morante, L. F. (2009): "La duración del plano a partir de la identificación de la información: referentes para la mejora del rendimiento del montaje”, Palabra Clave, vol. 12, no 1, pp. 153-164.

Pedrosa González, C. (2016): La estética y narrativa del vídeo musical como representante del discurso audiovisual hipermoderno (tesis doctoral). Madrid: Universidad Complutense de Madrid. Disponible en Internet: http://eprints.ucm. es/38085/ [Consultado el 07/07/2017].

Pérez Rufí, J. P. (2009): “El ritmo del spot de televisión actual. Narrativa audiovisual y categorías temporales en el palmarés del Festival Cannes Lions 2007". Zer. Revista de Estudios de Comunicación, vol. 14, no 27, pp. 103-124. Disponible en Internet: http://www.ehu.eus/ojs/index.php/Zer/article/view/2402 [Consultado el 07/07/2017].

Pérez Rufí, J. P., Navarrete Cardero, J. L., Gómez Pérez, F. J. (2014): “El product placement en el videoclip: análisis de contenido del emplazamiento de marca en los vídeos musicales preferidos por los usuarios de YouTube", Doxa Comunicación, no 18, pp. 83-104. Disponible en Internet: http://www.doxacomunicacion.es/es/hemeroteca/articulos?id=164 [Consultado el 07/07/2017]. 
Pérez Rufí, J. P y Rodríguez-López, J. (2017): “La duración del plano en el videoclip: hacia una categorización de los recursos formales en el vídeo musical contemporáneo", Zer. Revista de Estudios de Comunicación, vol. 22, no 42, pp. 335-52. Disponible en Internet: http://www.ehu.eus/ojs/index.php/Zer/article/view/17796 [Consultado el 07/07/2017].

Peverini, P. (2002): “Il videoclip: un’analisi dei dispositivi enunciativi”, en Pezzini, I. (ed.): Trailer, spot, clip, siti, banner. Le forme brevi della comunicazione audiovisiva. Roma: Melterni Editore, pp. 67-110.

Posner, M. (2016): “Mike Posner - I Took A Pill In Ibiza (Seeb Remix) (Explicit)”, YouTube. Internet: https://www.youtube. com/watch?v=foE1mO2yM04 [Consultado el 03/10/2017].

Rodríguez-López, J. (2016): "El vídeo musical como formato postmoderno: La ruptura de los códigos audiovisuales a través del clip”, Doxa Comunicación, no 21, pp. 13-30. Disponible en Internet: http://www.doxacomunicacion.es/es/hemeroteca/articulos?id=194 [Consultado el 07/07/2017].

Roig, A. y San Cornelio, G. (2015): "Prácticas de cocreación en vídeos musicales: el caso de Evolution of Get Lucky”, Anàlisi. Quaderns de Comunicació i Cultura, no 51, pp. 49-63. Disponible en Internet: www.raco.cat/index.php/Analisi/article/ download/304882/394690 [Consultado el 07/07/2017].

Sánchez Biosca, V. (1996): El montaje cinematográfico: teoría y análisis. Barcelona: Paidós.

Sedeño Valdellós, A. M. (2008): “La relación musicovisual en el videoclip: propuestas metodológicas y tipología”, en Aguilera Moyano, M., Adell I Pitarch, J. E. y Sedeño Valdellós, A. M. (eds.): Comunicación y música. Vol. 1. Barcelona: Editorial UOC, pp. 121-140.

- (2010) Sedeño Valdellós, A. M.: "Videoclips musicales en su transición a la red: nuevos subgéneros y apropiaciones del formato", Razón y Palabra, no 71. Disponible en Internet: http://www.razonypalabra.org.mx/N/N71/VARIA/25\%20SEDENO-REVISADO.pdf [Consultado el 07/07/2017].

Sedeño Valdellós, A. M., Rodríguez-López, J. y Roger-Acuña, S. (2016): “El videoclip postelevisivo actual. Propuesta metodológica y análisis estético", Revista Latina de Comunicación Social, nº 71, pp. 332-348. Disponible en Internet: www. revistalatinacs.org/071/paper/1098/RLCS-paper1098.pdf [Consultado el 07/07/2017].

Selva Ruiz, D. (2014): El videoclip. Comunicación comercial en la industria musical. Sevilla: Ediciones Alfar.

Tarín Cañadas, M. (2012): “La narrativa en el videoclip Knives Out, de Michel Gondry”, Icono 14, vol. 10, n. 2, pp. 148167. Disponible en Internet: http://www.icono14.net/ojs/index.php/iconol4/article/view/482/365 [Consultado el 07/07/2017]. 
- (2013): “El videoclip interactivo como nuevo formato en la red", en Lloves Sobrado, B. y Segado Boj, F. (ed.): I Congreso Internacional de Comunicación y Sociedad Digital. Logroño: Universidad Internacional de La Rioja. Disponible en Internet: https://dialnet.unirioja.es/servlet/articulo?codigo=4249528 [Consultado el 07/07/2017].

Vernallis, C. (2001): “The kindest cut: functions and meanings in the music video”. Screen, no 42, pp. 21-48.

- (2013): Unruly Media. YouTube, Music Video, and the New Digital Cinema. Oxford: Oxford University Press.

Villafañe Gallego, J. (2006): Introducción a la teoría de la imagen. Madrid: Pirámide.

Viñuela Suárez, E. (2008): “La autoría en el vídeo musical: signo de identidad y estrategia comercial”. Revista Garoza, n 8, pp. 235-247. Disponible en Internet: http://webs.ono.com/garoza/G8-Vinuela.pdf [Consultado el 07/07/2017]. 